Original Research

\title{
Time Course of Acute Hip Adduction Range of Motion Changes Following Foam Rolling
}

\author{
Anthony D'Amico ${ }^{1}$, Kevin Silva', Joseph Gallo ${ }^{1}$ \\ 1 Salem State University \\ Keywords: roller massage, non-contractile tissue, hip adduction, contractile tissue \\ https://doi.org/10.26603/001c.21417
}

\section{International Journal of Sports Physical Therapy}

Vol. 16, Issue 2, 2021

\section{Background}

With the increased popularity of foam rolling (FR), it is important to establish the exact manner in which the practice is useful.

\section{Purpose}

The purpose of this study was to examine the impact of FR the iliotibial (IT) band on hip adduction range of motion (ROM) and the short-term time course of any ROM changes that may occur.

\section{Method}

In a within-subject design, 34 subjects ( 21 female, 13 male) (female mean age $24.67 \pm 8.6$ yrs, height $161.4 \pm 9.8 \mathrm{~cm}$, mass $67.3 \pm 12.3 \mathrm{~kg}$; male mean age $22 \pm 2.5 \mathrm{yrs}$, height $170.2 \pm$ $8.2 \mathrm{~cm}$, mass $76.3 \pm 21.9 \mathrm{~kg}$ ) underwent a baseline Ober's test to measure hip adduction ROM prior to the FR and control conditions. Subjects rolled the lateral portion of each thigh for 3 bouts of 20 seconds. A tempo of 3 seconds down and 3 seconds up the leg was maintained across FR bouts. A 5-minute walk served as the control condition. The Ober's test was repeated at less than 1 minute, 3 minutes, 10 minutes, 15 minutes, and 20 minutes following the FR bouts and the control to assess changes in hip adduction ROM over time. A blinded clinician conducted the Ober's test. A two-way analysis of variance was used to assess differences by condition and time.

\section{Results}

No differences in hip adduction ROM were found at baseline between FR and CON within subjects $(27.9 \mathrm{~cm} \pm 7.5 \mathrm{vs} .27 .7 \mathrm{~cm} \pm 6.6, p>.05)$. Ober's test ROM was significantly greater in FR compared to CON immediately post-treatment $(24.2 \mathrm{~cm} \pm 6.3 \mathrm{vs} .28 .2 \mathrm{~cm} \pm 6.3, p=$ $.00, d=.59)$, and 3 minutes post $(24.6 \mathrm{~cm} \pm 7.6 \mathrm{vs} .28 .3 \mathrm{~cm} \pm 5.9, p=.00, d=.56)$. No differences were observed 10 minutes post, 15 minutes post, or 20 minutes post FR or $\mathrm{CON}(p>.05)$.

\section{Discussion}

Compared to walking, FR the IT band significantly increased hip adduction ROM as measured by the Ober's test. These increases appear to dissipate between 3 and 10 minutes post-FR.

\section{Conclusion}

An acute bout of 3 sets of 20 seconds of FR may be effective for transiently increasing

Corresponding Author:

Anthony D'Amico

Sport and Movement Science Department

Salem State University

352 Lafayette Street, Salem, MA 01970

978-542-2904 (phone)

978-542-6554 (fax)

adamico@salemstate.edu 
ROM. Whether these short-term increases have implications for chronic flexibility changes is unclear.

\section{Level of evidence}

\section{INTRODUCTION}

Foam rolling (FR) is an increasingly popular practice shown to improve range of motion (ROM) without concomitant decreases in strength and power. ${ }^{1,2}$ It is also thought to accelerate recovery from exercise-induced muscle damage. ${ }^{2,3}$ Foam rolling is commonly applied in therapeutic settings, with a purported benefit towards various soft tissue-based pathologies, though evidence supporting the use of FR for this purpose is limited. ${ }^{4}$

Investigations concerning FR's influence on acute flexibility changes typically report increases in between $4 \%$ and $16 \%{ }^{2}$ While these increases are smaller than those observed with static stretching (SS) alone, ${ }^{5}$ FR does not appear to acutely diminish strength and power, ${ }^{1}$ unlike long bouts of SS (>60 s). ${ }^{6}$ Most FR studies have assessed the ROM of tissues comprised of both contractile and non-contractile elements. ${ }^{7}$ Indeed, assessments of quadriceps, ${ }^{1}$ hamstrings, ${ }^{8}$ plantar flexors, 9,10 and other commonly-rolled muscle groups appear to indicate FR's efficacy in acute ROM increases. ${ }^{2}$ However, another commonly-rolled portion of the thigh, the iliotibial (IT) band, is not comprised of contractile tissue. It is a large, dense, fibrous portion of connective tissue located on the lateral aspect of the thigh. ${ }^{11}$ While the precise mechanisms underlying the ROM improvements associated with FR remain unclear, ${ }^{12}$ it is plausible that a largely fibrous tissue with less contractile elements will respond differently to treatment than one comprised largely of skeletal muscle. To the authors knowledge, only one study has explored FR applied to the non-contractile IT band, and no change in ROM was observed. ${ }^{13}$

Stretching, pain relief techniques, and other conservative treatments typically serve as the first line of therapy for IT band-related pathologies, ${ }^{11}$ such as IT band syndrome (ITBS). ${ }^{14}$ Iliotibial band syndrome is a common overuse injury often observed in runners and cyclists. Patients typically report lateral knee pain during repetitive lower-limb activities. ${ }^{14}$ Foam rolling has been shown to improve ROM ${ }^{1}$ and limited evidence suggests it may be effective for reducing sensations of pain. ${ }^{4,15-18}$ Though the efficacy for treating ITBS with FR has not been directly assessed, it is a common treatment in many rehabilitation settings. ${ }^{19}$ To date, the efficacy of FR as a therapeutic intervention for ITBS remains unclear. Patients with ITBS commonly demonstrate diminished hip adduction, ${ }^{11}$ possibly limited by the IT band's lack of extensibility. Thus, a treatment that could potentially improve hip adduction and reduce sensations of pain may offer value.

Clinical interventions that attempt to elongate the IT band have been widely used in physical rehabilitation. ${ }^{20}$ However, the capacity of the IT band to undergo elongation as a result of therapeutic interventions is an ongoing area of debate. ${ }^{20}$ Elongation interventions targeting the IT band, including the use of FR, have been recommended largely on the basis of clinical experience, and on the observation and measurement of increased hip adduction ROM after an intervention. ${ }^{20}$

Chaudhry et al. ${ }^{20}$ used a three-dimensional mathematical model for deformation of human fasciae in manual therapy to predict that a load of $9075 \mathrm{~N}(925 \mathrm{~kg})$ and a tangential force of $4515 \mathrm{~N}$ (460kg) would be required to produce even $1 \%$ compression and $1 \%$ shear. The authors noted that these predicted levels of forces are well beyond the physiologic range of manual therapy. Despite their findings, the authors speculated that mechanical stimulation of fascial mechanoreceptors may create changes in neural drive to skeletal muscle fibers connected to the IT band, which may explain the observed increases in hip adduction ROM seen in clinical practice following stretching, manual therapy, and FR. ${ }^{20}$ They further postulated that in vivo fascia may respond to mechanostimulation with an altered tonus regulation of its own. ${ }^{20}$

Empirical data supporting or refuting clinical observations of hip adduction improvements following FR may assist the practitioner's decision-making process. Additionally, an understanding of both the degree and duration of any change may be useful, as well. Despite acute ROM increases, limited evidence suggests a lack of chronic FR-induced improvements. ${ }^{21}$ Thus, at some point in time following FR, any improvements in ROM can be expected to dissipate. Previous investigations concerning contractile tissues have typically observed ROM increases lasting up to 10 minutes, with the effects fully dissipated at $30 \mathrm{~min}$ utes. $^{22}$ The duration of ROM changes following FR of noncontractile tissues has not been examined. This information could potentially benefit patient care, especially if FR is combined with other interventions directed at increasing ROM. The purpose of this study was to examine the impact of FR the IT band on hip adduction ROM and the short-term time course of any ROM changes that may occur. The hypothesis was that following FR of the IT band, hip adduction would increase compared to a control condition and that these increases would dissipate within twenty minutes.

\section{METHODS}

\section{SUBJECTS}

Subjects included 34 healthy adults (21 female, 13 male) (female mean age: $24.67 \pm 8.6 \mathrm{yrs}$, height $161.4 \pm 9.8 \mathrm{~cm}$, mass $67.3 \pm 12.3 \mathrm{~kg}$; male mean age $22 \pm 2.5 \mathrm{yrs}$, height $170.2 \pm 8.2 \mathrm{~cm}$, mass $76.3 \pm 21.9 \mathrm{~kg}$ ). Subjects were eligible for inclusion in the study if the medial joint line of the knee failed to reach the table during an Ober's test and if they had no lower extremity injury within the last six months. Prior to the first testing session, subjects provided informed consent as approved by the University Institutional Review Board. All procedures were approved by the University Institutional Review Board (IRB registration number: 00006274). Based upon previous research, 1,5,9,10,13 approx- 
imately 10 to 30 subjects per condition in a within-subject experimental design were determined as sufficient to observe a significant difference in the primary outcome measure of ROM.

\section{OBER'S TEST}

Ober's test is a clinical examination technique used to assess the extensibility of the IT band from its proximal origin at the tensor fascia lata to its distal insertion into the anterolateral tibia. ${ }^{23,24}$ The subject was positioned side-lying with the leg being tested on top, the bottom leg flexed for stability, and the hips stacked perpendicular to the table. The subject was positioned at the edge of the table to ensure that the lower leg and foot do not limit ROM. Positioned behind the subject, the examiner stabilized the pelvis proximally to maintain the stacked position for the hip to ensure proper lumbar and pelvic position. The examiner supported the lower leg distally at the anterior knee in order to limit potential hip internal or external rotation. By maintaining the hip in a neutral position with the distal hand around the anterior knee, the examiner was able to appreciate the end feel for the point of restriction as the subject's leg moves into adduction. The examiner passively brought the leg into abduction and extension before slowly releasing the lower limb toward the table with the knee flexed to approximately 10 degrees. The examiner identified the point of tissue restriction, which was indicated by restricted adduction of the thigh. In the present investigation, a second examiner was responsible for measuring the distance from the medial joint line to the top edge of the table in centimeters and provided additional visual observation to prevent any errors. The use of a tape measure to quantify the Ober's Test has been described by Doucette and Goble. ${ }^{25}$

\section{EXPERIMENTAL DESIGN}

A counterbalanced, repeated measures design was used to assess how FR the IT band influences hip adduction ROM and how long any changes were retained. Each subject completed two sessions on separate days; one where FR occurred and one where a 5-minute walk was performed as a control treatment, followed by a series of Ober's tests. Subjects were assigned to start with either the experimental or control condition in an alternating fashion to control for order effects. The dependent variable was hip adduction ROM as assessed by the Ober's test, specifically measured in $\mathrm{cm}$ between the medial joint line of the knee to the top of the treatment table. The independent variable was the condition, either FR prior to Ober's test measurements or not FR prior.

A baseline Ober's test was performed upon entry to the laboratory on both testing days. Figure 1 displays Ober's test, as it was performed in this investigation. Subjects then completed the FR protocol or the control treatment. When performing the FR protocol using a high-density foam roller (Thera-Band ${ }^{\circledR}$, Performance Health, Warrenville, IL, USA), subjects were instructed to roll the lateral portion of each thigh, from the greater trochanter to the lateral epicondyle of the knee. During FR, subjects were instructed to maintain a position that kept the lateral aspect of the thigh in contact

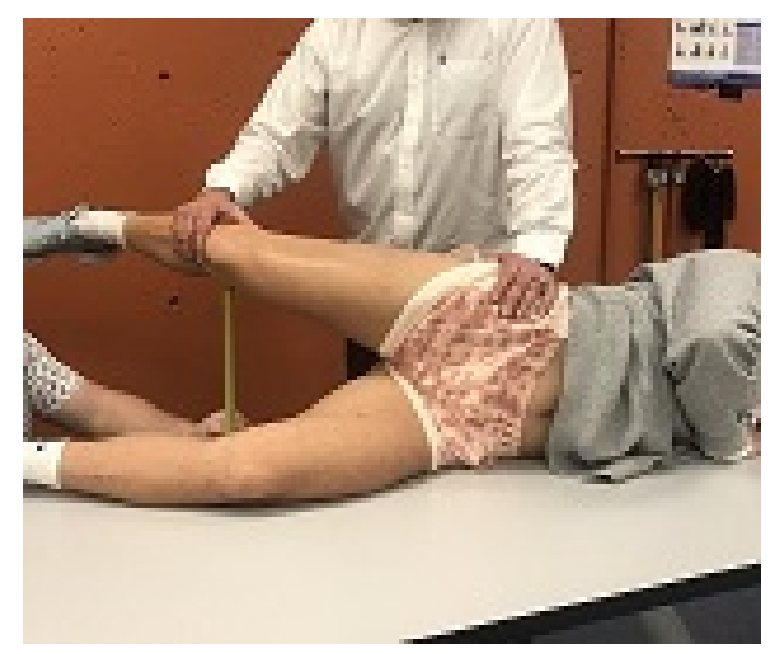

\section{Figure 1: Ober's test}

with the roller. A clinician, a licensed athletic trainer, observed the FR interventions and identified any substitutions and offered corrective feedback. The technique of FR from between the greater trochanter and the lateral epicondyle of the femur was employed to mimic typical clinical usage and guidelines. Subjects completed three 20-second FR bouts, each interspersed with a 20-second rest. Subjects were instructed to use a standardized FR technique by planting the foot and hands of the side opposite to that being rolled on the ground and shifting enough weight to those points so that pain did not exceed 6 on a scale of 10 . A tempo of 3 seconds down and 3 seconds up the leg was maintained across FR bouts.

Following the FR treatment or the 5-minute walk, the Ober's test was repeated immediately (less than 1 minute), 3 minutes, 10 minutes, 15 minutes, and 20 minutes to assess changes in hip adduction ROM over time. The Ober's test was performed by the same clinician across subjects. This clinician, a licensed physical therapist and athletic trainer, was blinded to the conditions and was solely responsible for conducting the Ober's test. The second clinician, a licensed athletic trainer, performed the measurement and observed for errors. The intrarater reliability of the Ober's test using a bubble inclinometer ranged from 0.91 and $0.94 .^{26}$ Intrarater reliability using a tape measure has not yet been reported in the literature.

\section{STATISTICAL ANALYSIS}

A two-way analysis of variance (ANOVA) was used to compare results for condition (FR vs. CON) across time (baseline, immediately, 3 minutes, 10 minutes, and 20 minutes post). Bonferroni post-hoc tests were conducted to compare replicate means by row. Alpha level was set at $p=0.05$. Cohen's $d$ effect sizes were calculated for all significant differences observed to determine the magnitude of effect. An effect size equal to or in excess of $0.2,0.5$, and 0.8 were considered small, moderate, and large effects, respectively. ${ }^{27}$ All data analysis was completed using GraphPad Prism 5.0 (GraphPad Software San Diego, CA, USA). 


\section{RESULTS}

Figure 2 displays changes in Ober test measurement over time following FR. Ober's test ROM was significantly greater in FR compared to CON immediately post-treatment $(24.2$ $\mathrm{cm} \pm 6.3$ vs. $28.2 \mathrm{~cm} \pm 6.3, p=0.00, d=0.59)$, with a moderate effect observed, and three minutes post $(24.6 \mathrm{~cm} \pm 7.6$ vs. $28.3 \mathrm{~cm} \pm 5.9, p=0.00, d=0.56$ ), with a moderate effect observed. No differences were observed between groups at baseline ( $27.9 \mathrm{~cm} \pm 7.5$ vs. $27.7 \mathrm{~cm} \pm 6.6, p>0.05, d=0.28)$ 10 minutes post $(26.4 \mathrm{~cm} \pm 5.4 \mathrm{vs} .27 .8 \mathrm{~cm} \pm 5.5, p>0.05, d$ $=0.26), 15$ minutes post $(27.4 \mathrm{~cm} \pm 6.3$ vs. $27.4 \mathrm{~cm} \pm 5.7, p>$ $0.05, d=0)$, or 20 minutes post FR or CON $(27.7 \mathrm{~cm} \pm 6.4 \mathrm{vs}$. $27.4 \mathrm{~cm} \pm 5.9, p>0.05, d=0.05)$.

\section{DISCUSSION}

The first objective of this study was to investigate the influence of FR the IT band on hip adduction ROM. The second objective of this study was to assess the short-term time course of any ROM changes observed following FR of the IT band. The first important finding in this study was that FR the IT Band for three bouts of 20 seconds increased hip adduction ROM as measured by the Ober's test. The second important finding in this study was that the observed increases in hip adduction ROM following FR dissipated sometime between three minutes and 10 minutes post-treatment. These findings indicate that 3 bouts of 20 seconds of FR can transiently increase ROM, even in a region primarily composed of non-contractile tissue. Further, the short-lived changes may have implications for the way FR is performed in both therapeutic and performance-oriented settings.

While transient increases in ROM are typically observed following FR, 1,5,9,10 the only other study assessing noncontractile tissue (also performed on the IT band) did not observe such a difference. ${ }^{13}$ Given the apparent similarities between that study and the present investigation, the reason for the contrasting findings is unclear. Hall and Smith ${ }^{13}$ instructed subjects to roll their IT band with an upright torso, while subjects in the present investigation positioned their torso parallel with the ground. While the former position may have decreased sensations of discomfort at the point of contact with the roller, Grabow et al. ${ }^{28}$ reported that higher rolling forces of the quadriceps did not amplify ROM increases. In the Hall and Smith ${ }^{13}$ study, subjects utilized a faster rolling cadence, transitioning up and down the length of the femur in two seconds, compared to the three seconds utilized in the present investigation. The influence of FR tempo on ROM has not been directly assessed, though it is plausible that slower tempos could result in greater increases. Another factor that may have influenced the differing outcomes of the present investigation and those of Hall and Smith ${ }^{13}$ could be the degree to which contractile tissues were influenced. In the present investigation, care was taken to focus on the non-contractile IT band to the greatest extent possible. Nevertheless, adjacent contractile tissues may have been influenced differently compared to the investigation by Hall and Smith, ${ }^{13}$ via altered exposure to the FR treatment. Subtle procedural differences between in-

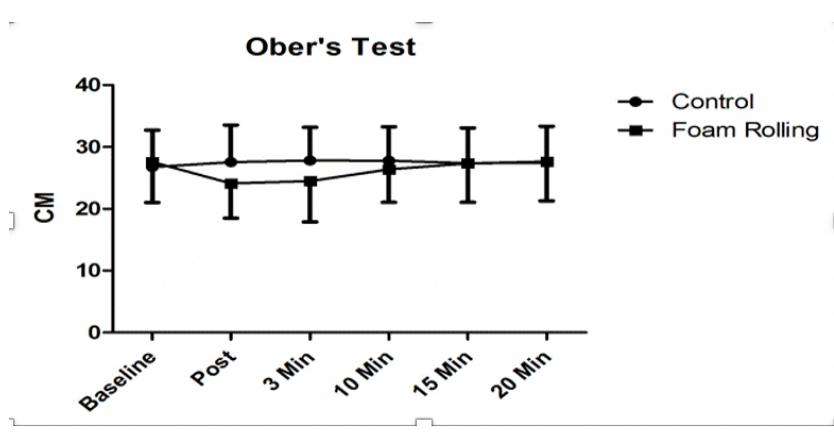

Figure 2: Mean change in Ober test range of motion following foam rolling or control. Significant difference by condition ( $p$ < 0.05 ) found immediately post-intervention and three minutes postintervention, with foam rolling resulting in greater range of motion.

vestigations will likely persist as a confounding variable in FR research, given the nature of the treatment.

To the authors' knowledge, the time course of changes in ROM following FR has only been investigated in conjunction with static stretching. ${ }^{22}$ While ROM was increased immediately post-treatment in that investigation, the effects had dissipated by 30 minutes. The quadriceps and hamstrings, two contractile muscle groups, were rolled in that investigation; therefore, similar outcomes might be expected in other contractile tissues. Based on the findings of the present investigation, increases in ROM may persist for at least three minutes after FR the IT band, with values returning to baseline some time before the 10-minute mark. While the increases in ROM observed in the present investigation reached statistical significance, they appear to be less pronounced than increases in trials assessing myofascial rolling treatment of contractile tissue. ${ }^{2}$ It stands to reason that while increases in ROM can potentially be attained by FR non-contractile tissues, the increases may be smaller and shorter-lived than those observed following treatment of contractile tissues.

The physiological underpinnings of ROM changes following FR are unclear, regardless of tissue type. There exists little consensus as to what specifically is modulated during treatment. Both local and global explanations have been put forth. ${ }^{29}$ Using acoustic radiation force imaging, Heiss et al. ${ }^{30}$ reported a $13 \%$ decrease in tissue stiffness of the IT band following FR. Despite the typically immediate and short-lived effects of FR, this decrease in stiffness was not apparent until 30 minutes after FR. Hotfiel et al. ${ }^{31}$ reported an increase in peak blood flow following FR of the lateral thigh, with increases of $73.6 \%$ immediately following FR and $52.7 \%$ at 30 minutes following FR. It is plausible that some combination of reductions in tissue stiffness and increases in local blood flow could explain the increased ROM observed following FR. In regards to potential non-local mechanisms, Behm and Wilke ${ }^{29}$ suggested that FR may activate global modulatory pain systems. This could potentially increase an individual's degree of stretch tolerance to that which may have been prohibitively uncomfortable prior to treatment. ${ }^{29}$ Non-local effects have indeed been evident in a number of investigations..$^{9,30-32}$ In some in- 
stances, joints contralateral to the one undergoing FR have increased ROM. ${ }^{9}$ Other findings include increased hamstring flexibility after FR of the plantar fascia, ${ }^{29}$ and hamstring FR influencing ROM in a region as remote as the shoulders. ${ }^{33}$ Additionally, Young et al. ${ }^{32}$ reported decreased Hoffman reflex responses after roller massage. These nonlocal effects would seem to confirm at least some degree of neural influence of FR.

In light of the non-local effects of FR, a favorable response to treatment for the IT band appears more plausible. Under a framework in which dense, rigid tissues needed to be mechanically altered for ROM to increase, successfully modifying the tension of the IT band with 60 cumulative seconds of FR would seem improbable. ${ }^{20}$ But considering the growing body of evidence that influential factors likely go beyond the mechanical, ${ }^{29}$ the findings of the present investigation are less surprising. While the approximate 3 to 10 minute time window of increased ROM may be limited to the IT band, the precise timeframe one can expect to maintain their increased ROM following FR warrants further exploration. The desired outcome of a FR session may dictate the importance of precise timing as well. If performed to expedite muscle recovery between training sessions or competitions, ${ }^{3,8}$ timing may be less critical. If FR is performed pre-training or pre-competition, timing may be immensely critical. For example, prior to a race, a hurdler may foam roll to increase ROM, eschewing static stretching for fear of decreasing muscular power and compromising sprinting speed. ${ }^{33}$ It is plausible that if FR is performed too long prior to competition, they will not reap the benefits. ${ }^{22}$ Similarly, in a therapeutic setting, a practitioner utilizing FR for a patient may stand to benefit knowing exactly how long they can expect any increases in ROM to persist. With that information, they may be able to functionally utilize that increased ROM within a therapy session.

This study had several limitations. First, the findings are limited to the rolling duration used. The influence of FR on hip adduction may be different if other lengths of treatment are used. Second, the IT band was the only area rolled. The influence of FR on ROM appears to vary considerably based on the specific area treated. Third, it is possible that nearby tissues such as the tensor fascia lata, vastus lateralis, and biceps femoris were influenced by the FR proto- col. Changes in those tissues may have impacted the results. Fourth, it is possible that the observed improvements in hip adduction ROM were not specifically due to FR, the IT band, or any specific tissue, but a global neurophysiological response that happened to be evident at the joint assessed. Foam rolling other areas with less obvious pertinence to hip adduction may result in similar outcomes. Fifth, given the intervals where data was collected, it cannot be determined where ROM was no longer increased after the threeminute mark. Sixth, intrarater reliability was not assessed for the clinicians performing and measured the Ober's Test using the tape measure methodology. Finally, subjects were not blinded in this study. The inability to blind subjects will likely remain a limitation in all FR investigations.

\section{CONCLUSIONS}

The findings of the present investigation support the hypothesis that FR applied to the IT band increases hip adduction ROM as measured by the Ober's test immediately post-treatment and at three minutes post-treatment. Foam rolling may be a useful tool for temporarily increasing hip adduction ROM. Practitioners should be aware that these changes are short-lived, and that a treatment intended to work in conjunction with increased hip adduction should transpire in a time-sensitive manner.

\section{ACKNOWLEDGMENTS}

These results were presented in an oral presentation at the European College of Sports Science Annual Congress in July 2018. No financial support was received.

\section{CONFLICT OF INTEREST STATEMENT}

The authors have no conflicts to report.

Submitted: April 09, 2020 CDT, Accepted: October 10, 2020

CDT 


\section{REFERENCES}

1. MacDonald GZ, Penney MDH, Mullaley ME, et al. An acute bout of self-myofascial release increases range of motion without a subsequent decrease in muscle activation or force. J Strength Cond Res. 2013;27(3):812-821. doi:10.1519/jsc.0b013e31825c2b c1

2. Beardsley C, Škarabot J. Effects of self-myofascial release: A systematic review. J Bodyw Mov Ther. 2015;19(4):747-758. doi:10.1016/j.jbmt.2015.08.007

3. Pearcey GEP, Bradbury-Squires DJ, Kawamoto J-E, Drinkwater EJ, Behm DG, Button DC. Foam rolling for delayed-onset muscle soreness and recovery of dynamic performance measures. J Athl Train. 2015;50(1):5-13. doi:10.4085/1062-6050-50.1.01

4. Wilke J, Vogt L, Banzer W. Immediate effects of self-myofascial release on latent trigger point sensitivity: A randomized, placebo-controlled trial. Biol Sport. 2018;35(4):349-354. doi:10.5114/biolspor t.2018.78055

5. Hodgson DD, Quigley PJ, Whitten JHD, Reid JC, Behm DG. Impact of 10-Minute interval roller massage on performance and active range of motion. J Strength Cond Res. 2019;33(6):1512-1523. doi:10.151 9/jsc.0000000000002271

6. Behm DG, Kibele A. Effects of differing intensities of static stretching on jump performance. Eur J Appl Physiol. 2007;101(5):587-594. doi:10.1007/s00421-00 7-0533-5

7. Kalichman L, Ben David C. Effect of self-myofascial release on myofascial pain, muscle flexibility, and strength: A narrative review. J Bodyw Mov Ther. 2017;21(2):446-451. doi:10.1016/j.jbmt.2016.11.006

8. Jay K, Sundstrup E, Søndergaard SD, et al. Specific and cross over effects of massage for muscle soreness: Randomized controlled trial. Int J Sports Phys Ther. 2014;9(1):82-91.

9. Kelly S, Beardsley C. Specific and cross-over effects of foam rolling on ankle dorsiflexion range of motion. Int J Sports Phys Ther. 2016;11(4):544-551.

10. Škarabot J, Beardsley C, Štirn I. Comparing the effects of self-myofascial release with static stretching on ankle range-of-motion in adolescent athletes. Int J Sports Phys Ther. 2015;10(2):203-212.

11. Beals C, Flanigan D. A review of treatments for iliotibial band syndrome in the athletic population. $J$ Sports Med. 2013;2013:367169. doi:10.1155/2013/367 169
12. Macgregor LJ, Fairweather MM, Bennett RM, Hunter AM. The effect of foam rolling for three consecutive days on muscular efficiency and range of motion. Sports Med Open. 2018;4(1):26. doi:10.1186/s 40798-018-0141-4

13. Hall M, Chadwick Smith J. The effects of an acute bout of foam rolling on hip range of motion on different tissues. Int J Sports Phys Ther. 2018;13(4):652-660. doi:10.26603/ijspt20180652

14. Strauss EJ, Kim S, Calcei JG, Park D. Iliotibial band syndrome: Evaluation and management. J Am Acad Orthop Surg. 2011;19(12):728-736. doi:10.5435/00124 635-201112000-00003

15. Aboodarda SJ, Spence AJ, Button DC. Pain pressure threshold of a muscle tender spot increases following local and non-local rolling massage. $B M C$ Musculoskelet Disord. 2015;16(1):265. doi:10.1186/s12 891-015-0729-5

16. Cavanaugh MT, Döweling A, Young JD, et al. An acute session of roller massage prolongs voluntary torque development and diminishes evoked pain. Eur J Appl Physiol. 2017;117(1):109-117. doi:10.1007/s004 21-016-3503-y

17. Macdonald GZ, Button DC, Drinkwater EJ, Behm DG. Foam rolling as a recovery tool after an intense bout of physical activity. Med Sci Sports Exerc. 2014;46(1):131-142. doi:10.1249/mss.0b013e3182a12 $\underline{3 d b}$

18. Pearcey GEP, Bradbury-Squires DJ, Kawamoto J-E, Drinkwater EJ, Behm DG, Button DC. Foam rolling for delayed-onset muscle soreness and recovery of dynamic performance measures. J Athl Train. 2015;50(1):5-13. doi:10.4085/1062-6050-50.1.01

19. Cheatham SW, Stull KR, Ambler-Wright T. Roller massage: Survey of physical therapy professionals and a commentary on clinical standards- part II. Int J Sports Phys Ther. 2018;13(5):920-930. doi:10.26603/ij spt20180920

20. Chaudhry H, Schleip R, Ji Z, Bukiet B, Maney M, Findley $\mathrm{T}$. Three-dimensional mathematical model for deformation of human fasciae in manual therapy. $J$ Am Osteopath Assoc. 2008;108(8):379-390. doi:10.755 6/jaoa.2008.108.8.379

21. Mohr AR, Long BC, Goad CL. Effect of foam rolling and static stretching on passive hip-flexion range of motion. J Sport Rehabil. 2014;23(4):296-299. doi:10.11 23/jsr.2013-0025 
22. Hodgson DD, Quigley PJ, Whitten JHD, Reid JC, Behm DG. Impact of 10-minute interval roller massage on performance and active range of motion. J Strength Cond Res. 2019;33(6):1512-1523. doi:10.151 9/jsc.0000000000002271

23. Reese NB, Bandy WD. Joint Range of Motion and Muscle Length Testing. Elsevier Health Sciences; 2016.

24. Reese NB, Bandy WD. Use of an inclinometer to measure flexibility of the iliotibial band using the Ober test and the modified Ober test: Differences in magnitude and reliability of measurements. J Orthop Sports Phys Ther. 2003;33(6):326-330. doi:10.2519/jos pt.2003.33.6.326

25. Douciette SA, Goble EM. The effect of exercise on patellar tracking in lateral patellar compression syndrome. Am J Sports Med. 1992;20(4):434-440. do i:10.1177/036354659202000412

26. Melchione WE, Sullivan MS. Reliability of measurements obtained by use of an instrument designed to indirectly measure iliotibial band length. J Orthop Sports Phys Ther. 1993;18(3):511-515. doi:1 $\underline{0.2519 / \text { jospt.1993.18.3.511 }}$

27. Fritz CO, Morris PE, Richler JJ. Effect size estimates: Current use, calculations, and interpretation. J Exp Psychol Gen. 2012;141(1):2-18. $\underline{\mathrm{d}}$ oi: $10.1037 / \mathrm{a} 0024338$
28. Grabow L, Young JD, Alcock LR, et al. Higher quadriceps roller massage forces do not amplify range-of-motion increases nor impair strength and jump performance. I Strength Cond Res.

2018;32(11):3059-3069. doi:10.1519/jsc.00000000000 $\underline{01906}$

29. Behm DG, Wilke J. Do self-myofascial release devices release myofascia? Rolling mechanisms: A narrative review. Sports Med. 2019;49(8):1173-1181. $\underline{\mathrm{d}}$ oi:10.1007/s40279-019-01149-y

30. Heiss R, Mayer I, Huettel M, et al. Evaluation of tissue stiffness in athletes with different experience in foam rolling assessed by acoustic radiation force impulse elastography. Semin Musculoskel Radiol. 2019;23:1-6. doi:10.1055/s-0039-1687708

31. Hotfiel T, Swoboda B, Krinner S, et al. Acute effects of lateral thigh foam rolling on arterial tissue perfusion determined by spectral doppler and power doppler ultrasound. J Strength Cond Res. 2017;31(4):893-900. doi:10.1519/jsc.00000000000016 41

32. Young JD, Spence A-J, Behm DG. Roller massage decreases spinal excitability to the soleus. J Appl Physiol. 2018;124(4):950-959. doi:10.1152/japplphysi ol.00732.2017

33. Monteiro ER, Costa PB, Corrêa Neto VG, Hoogenboom BJ, Steele J, da Silva Novaes J. Posterior thigh foam rolling increases knee extension fatigue and passive shoulder range-of-motion. J Strength Cond Res. 2019;33(4):987-994. doi:10.1519/jsc.000000 0000003077 